\title{
Rhabdomyosarcoma discovered in the adrenal region of an elderly hypertensive patient
}

\author{
Hypertension Research (2011) 34, 784-786; doi:10.1038/hr.2011.29; published online 17 March 2011
}

Rhabdomyosarcoma is rare soft-tissue tumor that is more prevalent among children. In contrast, rhabdomyosarcomas are extremely rare in adults, with soft-tissue sarcomas making up $<1 \%$ of malignancies in adults and rhabdomyosarcomas accounting for $<2 \%$ of all soft-tissue sarcomas. ${ }^{1}$ Rhabdomyosarcoma in the adrenal region has not been documented to date. We here report a very rare case of rhabdomyosarcoma occurred in the left adrenal region of an elderly hypertensive patient.

A 76-year-old Japanese woman with a 5-year history of hypertension (occasionally systolic blood pressure $>200 \mathrm{~mm} \mathrm{Hg}$ ) was referred to our hospital. The patient had complained about left flank pain for 2 months. Abdominal computed tomography detected a left adrenal mass of $7 \mathrm{~cm}$ in diameter (10-40 Hounsfield units) heterogeneously contrasted at the early enhancement (Figure 1). The tumor exhibited a heterogeneous echo with regular surface by ultrasound. Magnetic resonance imaging exhibited a diffusely low-intensified adrenal mass by T1-weighted images. T2-hyperintensity was spottily detected in the central area
(Figure 1). Fat-suppression magnetic resonance imaging showed the least inclusion of adipose tissue in the tumor. Scintigram with ${ }^{131}$ I-adosterol showed almost normal pattern of bilateral uptake in the adrenal regions. Peripheral blood cell count, liver and renal functions, blood glucose and electrolytes were normal. The basal endocrine data of adrenal functions were within normal ranges as follows: plasma adrenocorticotropin, $29.2 \mathrm{pg} \mathrm{ml}^{-1}$ (normal: 7-55); cortisol, $10.6 \mu \mathrm{g} \mathrm{dl}^{-1}$ (8-25) with normal circadian fluctuation and significant suppression $\left(<0.5 \mu \mathrm{g} \mathrm{dl}^{-1}\right)$ after $1 \mathrm{mg}$
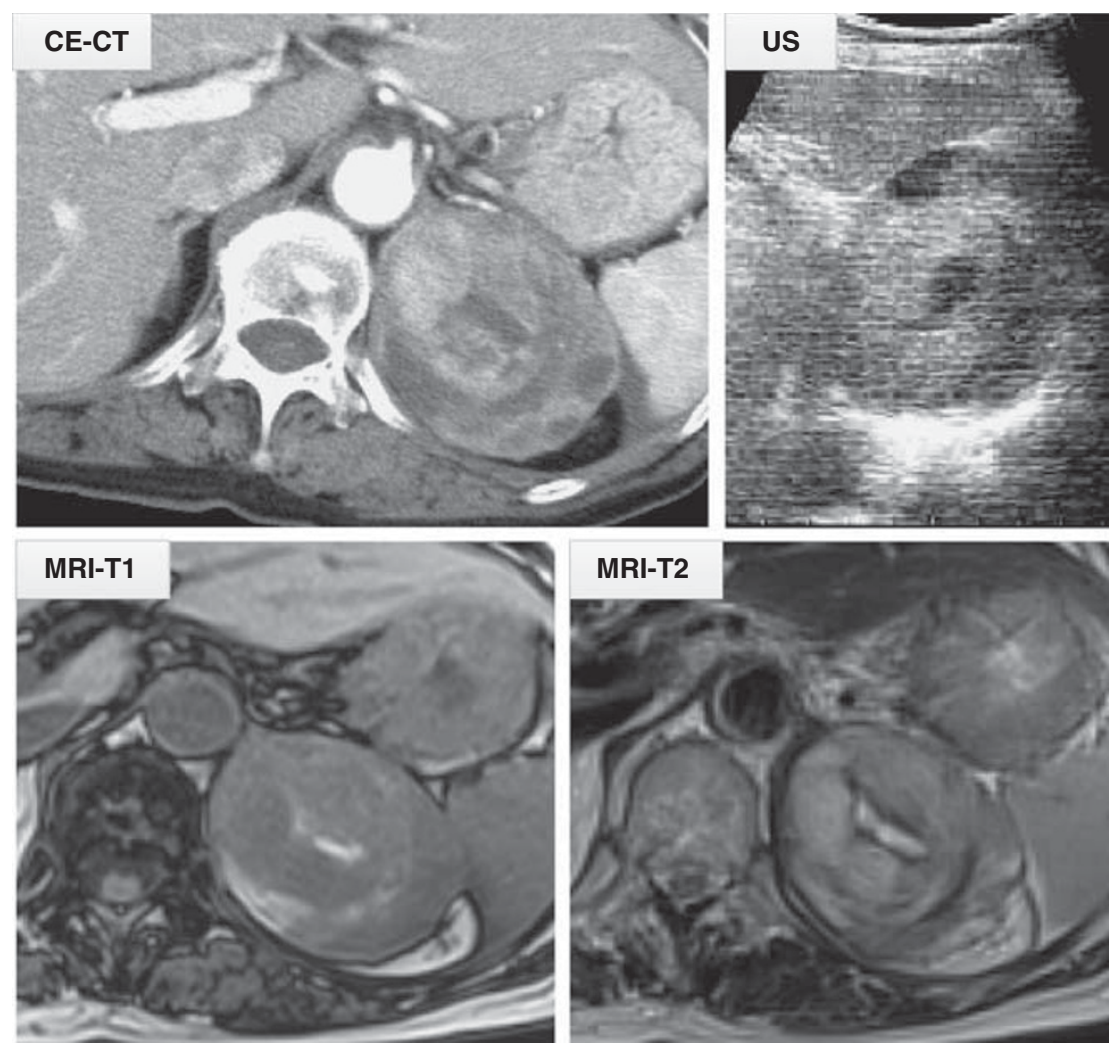

Figure 1 Radiological findings of the left adrenal tumor. Enhanced computed tomography scan (CE-CT), ultrasonography (US), and T1- and T2-weighted magnetic resonance images (MRI) are shown. 


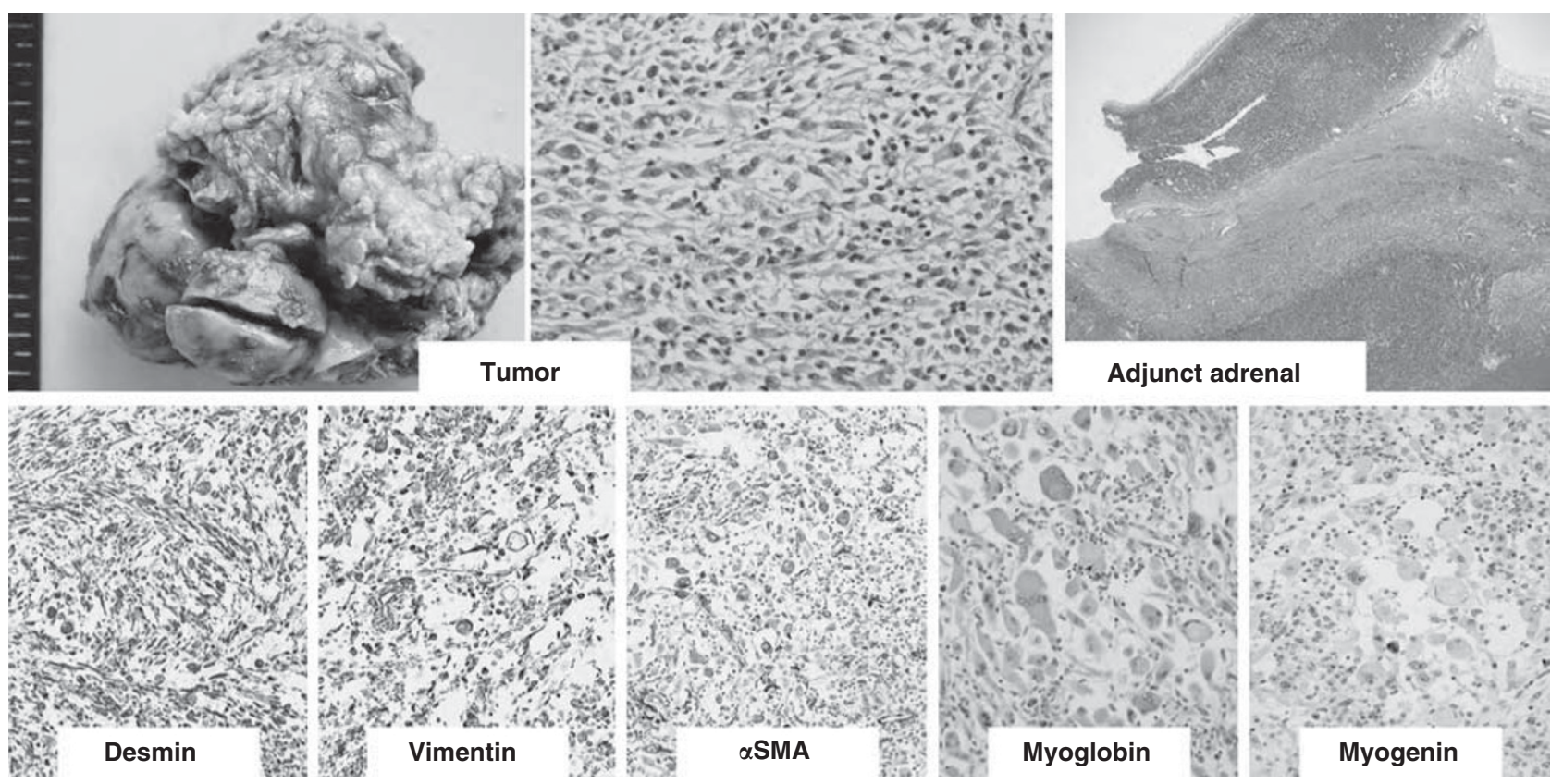

Figure 2 Pathological findings of the resected tumor. The gross feature and histological findings of the resected tumor $(\times 400)$ and the adjunct normal adrenal tissue $(\times 40)$ are shown by hematoxylin-eosin staining. Tumor sections are immunopositive with desmin, vimentin, $\alpha$-smooth muscle actin ( $\alpha$ SMA), myoglobin and myogenin staining.

oral dexamethasone administration; plasma renin activity, $0.3 \mathrm{ng} \mathrm{ml}^{-1} \mathrm{~h}^{-1}(0.2-2.7)$; aldosterone, $4.3 \mathrm{ng} \mathrm{dl}^{-1}$ (3.6-24); dehydroepiandrosterone sulfate, $42 \mu \mathrm{g} \mathrm{dl}^{-1}$ (13-154). Urinary excretion of catecholamines was normal, with urinary adrenaline, $4.5 \mu \mathrm{g}$ per day (3-41); noradrenaline, $94.1 \mu \mathrm{g}$ per day (31-160); dopamine, $434.5 \mu \mathrm{g}$ per day (280-1100); metanephrine, $0.06 \mathrm{mg}$ per day (0.04-0.18); normetanephrine, $0.23 \mathrm{mg}$ per day $(0.10-0.28)$; and vanillyl mandelic acid, $2.5 \mathrm{mg}$ per day (1.5-4.3). Upon diagnosis of nonfunctioning adrenal malignancy, surgical resection of left adrenal tumor was attempted. Before surgery the patient's blood pressure was adequately controlled to $112 / 74 \mathrm{~mm} \mathrm{Hg}$ by amlodipine with salt restriction. In the surgical procedure, the left adrenal tumor had tightly adhered to the diaphragm, liver and spleen. The resected adrenal tumor was composed of grayish rubbery tissues with degeneration due to necrosis and hemorrhage (Figure 2). The tumor was microscopically separated from the renal parenchyma by a renal fibrous capsule. Residual adrenal tissues were not apparently atrophic but stretched over the surface of the tumor (Figure 2). Pathological evaluation of the tumor revealed interlacing fascicles of pleomorphic spindle cells showing mitotic rate as high as 2 out of 10 cells in high-power field (HPF). The composed cells were positive with desmin, myoglobin, myogenin, vimentin and $\alpha$-smooth muscle actin staining, whereas those were negative with cytokeratin AE1/AE3, cytokeratin CAM5.2, cal- desmon, S100 protein, inhibin- $\alpha$ and calretinin (Figure 2). These indicated that the tumor was derived from rhabdomyocytes, leading to the diagnosis of pleomorphic-type rhabdomyosarcoma. After the tumor removal, concurrent hypertension was readily ameliorated. Recurrence or metastasis of the primary tumor has not been detected for 2 years.

According to a recent review by Stock et $a l^{2}$ with respect to adult-type rhabdomyosarcoma, the tumors were mainly located in the lower limbs (47\%), trunk walls (26\%) and upper limbs (18\%). The tumors developed exclusively in deep soft tissues (94\%) and often involved the striated muscle. The sizes of the tumors ranged from 1 to $30 \mathrm{~cm}$ and $71 \%$ of the cases were $>5 \mathrm{~cm}$. Only two cases of rhabdomyosarcomas were reported in the retroperitoneal region; however, the cases in the adrenal region have not been reported.

It is extremely difficult to diagnose rhabdomyosarcoma as an adrenal incidentaloma preoperatively. In the radiological aspect, ${ }^{3}$ the tumors were either isodense or slightly hyperdense to skeletal muscle by computed tomography. On T1-weighted images of magnetic resonance imaging, 81\% rhabdomyosarcoma cases were isointense to skeletal muscle and $19 \%$ were slightly hyperintense. On T2weighted images, 69\% rhabdomyosarcomas were moderately hyperintense and $32 \%$ were strongly hyperintense, in which pleomorphic tumors had very high and heterogeneous signals as seen in our case. However, active or recent hemorrhage and calcification were not found in any of the cases. In the pathological aspect, adult-type rhabdomyosarcomas are diagnosed according to morphologic criteria as well as positive immmunostaining for desmin and/or myoglobin, which are filamentous and oxidationrelated antigens. ${ }^{2,4}$ Intranuclear transcription factors called myogenin and MyoD1 are also sensitive and specific markers for striated muscle differentiation and, as expressed in the present case, it is now well established that the positivity of one of these markers is necessary for diagnosis of rhabdomyosarcoma. $^{5-7}$ As for the prognosis, ${ }^{2} 25 \%$ of adult-type rhabdomyosarcoma patients died of cancer or therapeutic complications. The overall 5-year survival and metastasis-free survival rates were 53 and $63 \%$, respectively. In addition, adult cases with rhabdomyosarcoma had significantly worse outcome than children (5-year survival rates, 27 and 61\%, respectively). ${ }^{8}$ Multivariate analysis showed that aged, pleomorphic and alveolar subtypes, unfavorable location, advanced disease stage, and loss of local control with surgery and/or radiation were significant predictors of worse survival.

Although rhabdomyosarcomas originate from skeletal muscle, the vast majority occurs in the extremities and head/neck region and less commonly on the trunkal, intrathoracic and retroperitoneal regions. In the present 
case, we presume that the tumor was derived from diaphragm and/or iliopsoas muscles, according to the surgical finding that the tumor was tightly adhered to the diaphragm. The reduction of blood pressure after surgery also implies the possibility that adrenal rhabdomyosarcoma is, at least in part, related to the subclinical activation of sympathetic nerve activity and/or adrenal hormone release. In this regard, an adult female hypertensive case having renal rhabdomyosarcoma was reported, ${ }^{9}$ in which plasma renin activity was preoperatively high possibly due to the intrarenal vessel compression. In our case, the alteration of adrenal hormones was not observed before and after surgery. It is still possible that sympathetic nervous activity and/or other humoral factors associated with rhabdomyosarcoma could be involved in the patient's concurrent hypertension. The possibility of sarcoma should also be kept in mind in cases of adrenal incidentalomas with enlarged and heterogenous radiological findings. Complete surgical resection without microscopic residue is important for the better prognosis.
Akihiro Katayama $^{1}$, Fumio Otsuka ${ }^{1}$, Masaya Takeda ${ }^{1}$, Tomoko Miyoshi ${ }^{1}$,

Eri Nakamura ${ }^{1}$, Kenichi Inagaki ${ }^{1}$,

Takehiro Tanaka ${ }^{2}$, Shinya Uehara ${ }^{3}$ and Hirofumi Makino ${ }^{1}$

${ }^{1}$ Department of Medicine and Clinical Sciences, Okayama University Graduate School of Medicine, Dentistry and Pharmaceutical Sciences, Okayama, Japan; ${ }^{2}$ Department of Pathology, Okayama University Graduate School of Medicine, Dentistry and Pharmaceutical Sciences, Okayama, Japan and ${ }^{3}$ Department of Urology, Okayama University Graduate School of Medicine, Dentistry and Pharmaceutical Sciences, Okayama, Japan E-mail: fumiotsu@md.okayama-u.ac.jp

1 Hawkins WG, Hoos A, Antonescu CR, Urist MJ, Leung DH, Gold JS, Woodruff JM, Lewis JJ, Brennan MF. Clinicopathologic analysis of patients with adult rhabdomyosarcoma. Cancer 2001; 91: 794-803.

2 Stock N, Chibon F, Binh MB, Terrier P, Michels JJ, Valo I, Robin YM, Guillou L, Ranchere-Vince D, Decouvelaere AV, Collin F, Birtwisle-Peyrottes I, Gregoire F,
Aurias A, Coindre JM. Adult-type rhabdomyosarcoma: analysis of 57 cases with clinicopathologic description, identification of 3 morphologic patterns and prognosis. Am J Surg Pathol 2009; 33: 1850-1859.

3 Allen SD, Moskovic EC, Fisher C, Thomas JM. Adult rhabdomyosarcoma: cross-sectional imaging findings including histopathologic correlation. Am J Roentgenol 2007; 189: 371-377.

4 Furlong MA, Mentzel T, Fanburg-Smith JC. Pleomorphic rhabdomyosarcoma in adults: a clinicopathologic study of 38 cases with emphasis on morphologic variants and recent skeletal muscle-specific markers. Mod Pathol 2001; 14: 595-603.

5 Kumar S, Perlman E, Harris CA, Raffeld M, Tsokos M. Myogenin is a specific marker for rhabdomyosarcoma: an immunohistochemical study in paraffin-embedded tissues. Mod Pathol 2000; 13: 988-993.

6 Cessna MH, Zhou H, Perkins SL, Tripp SR, Layfield L, Daines C, Coffin CM. Are myogenin and myoD1 expression specific for rhabdomyosarcoma? A study of 150 cases, with emphasis on spindle cell mimics. Am J Surg Pathol 2001; 25: 1150-1157.

7 Morotti RA, Nicol KK, Parham DM, Teot LA, Moore J, Hayes J, Meyer W, Qualman SJ. An immunohistochemical algorithm to facilitate diagnosis and subtyping of rhabdomyosarcoma: the Children's Oncology Group experience. Am J Surg Pathol 2006; 30: 962968

8 Sultan I, Qaddoumi I, Yaser S, Rodriguez-Galindo C, Ferrari A. Comparing adult and pediatric rhabdomyosarcoma in the surveillance, epidemiology and end results program, 1973 to 2005: an analysis of 2,600 patients. J Clin Oncol 2009; 27: 3391-3397.

9 Selvaggi FP, Fabiano G, Santacroce S. Hypertensive renal rhabdomyosarcoma. Eur Urol 1979; 5: 371-372. 